\title{
Drag in the city : Eléments pour une analyse du paysage Drag Queen français
}

Arnaud Alessandrin : Sociologue à l'université de Bordeaux (LACES, 7437). Ses travaux portent sur les questions de genre, de santé et de discriminations. II est l'auteur de " Sociologie des transidentités" (Cavalier Bleu, 2018) et de "Fan studies - Gender studies : le retour » avec Mélanie Bourdaa (Téraèdre, 2019).

Résumé : A travers des entretiens avec des Drag Queens françaises, cet article se propose de revenir sur les formes contemporaines de la pratique Drag en France. L'émergence de la scène drag actuelle, qui fait suite aux figures connues des années 1990, est interprétée à l'aune d'entretiens auprès de 20 dragqueens. Ces derniers s'attachent à mettre en lumière les questions variées qui innervent la scène drag actuelle comme la culture queer, l'homophobie, le féminisme, la précarité ainsi que l'importance des représentations écraniques qui poussent aujourd'hui les figures Drag du côté d'une visibilité jamais atteinte dans la sphère publique.

Mots clés : Drag Queens, Genre, LGBTphobie.

\section{Introduction : sur les traces d'un « renouvellement » Drag}

Dans les années 1990, comme dans le reste du monde, la France connaît un essor de la visibilité Drag (Schacht et Underwood, 2003). Jusque-là, le personnage de Divine, popularisé par les films de John Waters ${ }^{1}$, tenait pour unique représentation populaire des Drag Queens. Longtemps assimilées aux espaces du travestissement et du cabaret (Foerster, 2012), aux cultures $\mathrm{LGBT}^{2}$ et à la vie nocturne, plusieurs figures apparaissent, dans une temporalité relativement courte. Le grand écran et la chanson sont alors le théâtre de quelques apparitions remarquées ${ }^{3}$. En 1990 , le documentaire "Paris is burning " ${ }^{4}$ restitue la naissance du "voguing", un type de danse urbaine qui prend racine dans la communauté homosexuelle et noire des Etats-Unis (Bressin et Patinier, 2012) et filme des Drags de Harlem participant à un concours de Drag Queens. Devenu culte, ce documentaire n'est pourtant pas la première visibilité Drag au cinéma. Comme le souligne Didier Roth-Bettoni (2007), "l'irruption du phénomène Drag Queens" (p.343) s'accompagne de films moins connus, comme "Aliens cuts my hair » de Michael McIntosh en 1992 ou bien encore de "Vegas in space » de Philip R. Ford la même année. Mais c'est bel et bien « Paris is burning » qui sera retenu par la culture LGBT comme un initiateur des figures Drag en cette décennie.

\footnotetext{
${ }^{1}$ On pensera notamment au film « Pink Flamingos » (1972).

${ }^{2}$ LGBT : Lesbiennes Gays Bisexuel.le.s et Trans.

${ }^{3}$ Restituons avec un peu plus de précision ce qui se déroule avant les années 1990 : les Drag ne disparaissent pas et des films comme "Torch Song Trilogy » (1988), "Victor Victoria » (1982), " The Rocky Horror Picture Show » (1975) inscrivent des figures Drag dans la culture cinématographique -avec des amalgames certains avec les transidentités.

${ }^{4}$ Film de Jennie Livingston.
} 
Toutefois, c'est très certainement le film "Priscilla folle du désert ${ }^{5}$ qui, en 1994, sera le point culminant de la visibilité Drag $^{6}$. En parallèle de cela, la télévision prend une part active dans la mise en lumière de ces créatures spectaculaires et sophistiquées. Certes plus timide que les Etats Unis en matière de production documentaire sur cette question (Roth Betoni, 2007), les chaînes publiques françaises accordent quelques reportages au phénomène. Entre autre moments forts, on doit à l'émission « Envoyé spécial » un reportage entièrement dédié à " L'univers Drag Queen » en $1995^{7}$ ainsi qu'aux actualités régionales d'île de France un reportage sur "la première agence de Drag Queen créée en France, à Paris » qui propose une " meilleure gestion et protection des Drags Queen et de leur carrière " ${ }^{8}$. Du côté de la musique également, la figure Drag Queen connaît une visibilité jamais atteinte en France. En 1995 le groupe "Sister Queen " sort le single "Let me be a Drag Queen » qui se hisse jusqu'à la neuvième place des ventes de singles. Invités sur de nombreux plateaux télévisés, les membres du groupe arborent ce qui deviendra le symbole Drag des années 1990 : une perruque colorée et surdimensionnée, associée à des chaussures plateformes tout aussi extravagantes. Enfin, pour ne citer que quelques évènements médiatiques marquants, l'année 1996 offre un autre instant de visibilité aux Drag Queens lors de la tournée "Tour 96 » de la chanteuse Mylene Farmer qui accompagne sa chanson "Sans contrefaçon » de quatre danseurs Drag Queenés sur scène. Entre 1990 et 1996, la figure Drag Queen s'expose. Pour autant, la culture Drag Queen, celle de la performance singulière (Greco, 2016), du personnage scénique, celle aussi de la "communauté " propre aux Drag ne parvient pas à s'installer (Edward et Farrier, 2020). Ce qui demeure, c'est l'extravagance des personnages, tous un peu similaires à vrai dire, durant ces quelques années sous les lumières médiatiques.

Pourtant, en 2009, un autre évènement médiatique majeur va remettre les Drag Queens sous les feux des projecteurs. RuPaul, Drag Queen américaine, lance le pari d'une émission de télé-réalité ayant pour principe un concours de Drag Queens. Son nom : le "RuPaul's Drag Race ». A chaque saison est élue une "american's next Drag superstar " qui, à son tour, popularise la pratique Drag. La renaissance de la culture Drag est immédiate et la France n'est pas épargnée par le phénomène.

Cependant, d'un point de vue sociologique, il n'est pas évident que les expériences et les pratiques que nous pouvons décrire aujourd'hui soient les mêmes que celles qui ont traversées les années 1990. A travers vingt des entretiens réalisés entre 2017 et 2019, cet article porte un regard réactualisé sur une contre-culture du genre et se donne pour ambition de restituer quelques glissements subjectifs et politiques des Drag dans un contexte français $^{10}$. C'est donc simultanément d'engagements, d'émotions et de

\footnotetext{
${ }^{5}$ Film de Stephan Elliott.

${ }^{6}$ En 1995, le film «Extravagances» de Beeban Kidron reprend des figures proches à celles de Priscilla et donnent à voir un Road-Movie qui montre l'influence du film d'Elliott durant cette décennie.

7 "L'univers Drag Queen », reportage de Michel Mompontet pour l'émission Envoyé Spécial du 12 octobre 1995. France 2.

${ }^{8}$ Reportage du 12 mars 1996, réalisé par Xavier Collombier. Actualité régionales île de France, France 3 Paris (01min51). Les citations proviennent du descriptif INA - Institut National de l'Audiovisuel - relatif à cette émission.

${ }^{9}$ La prochaine superstar Drag des Etats-Unis.

${ }^{10}$ Tout au long de cet article, ni les noms des interviewées ni même leurs noms de scène ne seront dévoilés.
} 
représentations dont il s'agit ici. Au travers des témoignages recueillis, nous interrogerons certains éléments matériels et représentationnels qui reviennent dans les pratiques des Drag rencontrées. Cet article se limite toutefois à la parole de Drag Queens et n'aborde pas son corolaire, beaucoup plus invisible médiatiquement comme sociologiquement, de la pratique Drag King (Greco, 2014; Alessandrin; 2014). Enfin, le recrutement des personnes interrogées ne prend pas en considération la fréquence de la pratique, même si, pour l'immense majorité d'entre elles, la pratique Drag était assez nettement régulière, voire hebdomadaire. Une typologie variée de Drag Queens a ainsi été prise en compte (Alessandrin, 2021 ; Patinier, 2014) même si les profils sociodémographiques rencontrés sont cependant similaires sur de nombreux points : toutes les Drag ont entre 18 et 35ans, à l'exception d'une seule de plus de 40 ans $^{11}$. Elles sont toutes de sexe masculin et se déclarent homosexuelles, et toutes se considèrent, en dehors de la scène, comme des personnes cisgenres $^{12}$. Le recrutement des interviewées s'est effectué «au fil des rencontres " en débutant par deux lieux préalablement ciblés (sur Paris et Bordeaux), et ouverts à l'enquête grâce à des Drag qui nous ont permis de nous introduire dans ce milieu. Les entretiens, bien souvent prévus afin de pouvoir enregistrer, se sont pour la moitié d'entre eux déroulés de façon formelle et informelle (lors d'invitations à des shows par exemple). Le contexte des récits modifie donc fatalement les témoignages car l'immersion dans les salles des représentations donne lieu à des questions moins théoriques de la part du chercheur, et à des réponses bien plus spontanées de la part des interviewées. Au travers des thématiques comme celle de la politisation du Drag, de la précarité, de la nouvelle médiatisation du phénomène ou bien encore de l'explosion de " house ${ }^{13}, c^{\prime}$ est-à-dire de collectifs de Drag, cet article retrace donc deux années d'entretiens auprès de Drag aussi bien parisiennes qu'habitant en province. Quelles expériences, représentations et engagements sont communs, ou à l'inverse distants, entre les années 1990 et aujourd'hui en ce qui concerne la scène Drag française ? Par la même, peut-on dire que la pratique Drag est-elle une forme de politisation aujourd'hui qui va au-delà de la déconstruction de genre ? Dans une perspective panoramique (autour de plusieurs thématiques) et comparative (entre deux décennies clés) cet article tente également de renouer avec l'analyse d'une figure de la subversion de genre un peu oubliée.

\section{$I^{\circ}$ « Vraie » ou « Fausse » Drag : I’enjeu politique au cœur de la légitimité Drag.}

B. est une Drag "des années 90 » comme elle se définit. A 42 ans elle assume son âge et l'expérience qui va avec: "il n'y en a plus beaucoup des comme-moi. Je suis un dinosaure » dit-elle. Dans l'entretien qu'elle m'accord, B. insiste longuement sur le glissement politique qui accompagne les mouvements Drag, et en particulier ceux des années 1990 et 2010.

\footnotetext{
${ }^{11}$ Il est important de souligner un angle mort majeur que ce panel d'âge. En interrogeant massivement des jeunes Drag, nous avons accès aux représentations de cette génération, mais il est impossible de confirmer ces dernières en l'absence d'entretiens (sinon un) de Drag plus âgées, ayant pratiqué le Drag dans les années 1990 par exemple.

${ }^{12}$ C'est-à-dire dont l'identité de genre correspond au sexe assignée à la naissance.

${ }^{13}$ Le terme de " House " renvoie non seulement à l'idée d'une " maison " c'est-à-dire d'une solidarité clanique mais aussi à l'idée d'une culture propre à chaque regroupement (Bressin et Patinier, 2014).
} 
"Attention vous allez voir le logo ORTF apparaître, mais c'est vrai que les Drags d'aujourd'hui n'ont pas connu le sida comme nous. C'est super évidemment, mais pour des vieilles telles que moi, ça nous force à changer de logiciel. Passer des VHS à Twitter en quelque sorte ". Comme le soulignent Tiphaine Bressin et Jérémy Patinier dans leurs travaux sur le voguing, les années 1990 impriment sur le mouvement Drag des thématiques centrales comme celles de la lutte contre le VIH. C'est également ce que rappelle Jean Yves le Talec dans son ouvrage consacré aux " sœurs de la perpétuelle indulgence ", groupe de personnes travestis en nonnes et luttant, par la parodie et l'activisme, contre le VIH et les IST (Le Talec, 2008). Cette transformation flagrante des thématiques de la lutte nous indiquent que la pratique du Drag et le contexte politique d'expression sont sans cesse reliés. "Etre une Drag Queen c'est être dans la cité chérie. On fait pas tout ça pour se cacher. On bouscule et c'est tant mieux. On tape là où ça fait mal » dit G., Drag depuis 7ans et qui vit à Paris. G. "n'en veut pas aux Drag Queens des années 90 » mais estime néanmoins que " c'est pas tout d'être visibles, il faut avoir un truc à dire. Danser sur Mylene Farmer ça n'a rien de dégradant mais j'ambitionne autre chose avec mes sœurs ${ }^{14}$ ". Si les registres de la politisation se déplacent en fonction des thématiques mises en avant par les mouvements sociaux et les politiques LGBT, la question de la légitimité Drag semble aujourd'hui passer par une politisation ${ }^{15}$ du discours et de la pratique ${ }^{16}$ qui s'appuie sur de nouveaux référentiels. Pour A. "La théorie Queer est constamment présente dans notre pratique ". Elle insiste : " C'est hyper important d'avoir un discours construit et des livres comme ceux de Judith Butler ou Sam Bourcier ont été de vraies révélations pour moi ". S'arrimer à la théorie pour proposer un univers scénique revient, pour le dire comme Eric Fassin à un " usage de la science " (2005), une réappropriation ainsi qu'une politisation; une " pratique de la théorie " (Perreau, 2018) qui s'inscrit très nettement dans les pas des écrivaines et écrivains queers et de certains courants féministes (Shaffauser, 2014). B. de B. relate elle aussi cette politisation progressive dans son parcours : "Au début je faisais vraiment ça pour m'amuser, pour créer un personnage. J'adorai ça. Et puis un jour j'ai rencontré J., une Mother Drag ${ }^{17}$ devenue célèbre sur Paris. Elle nous a coupé net dans notre élan de gamines en nous mettant entre les mains du Butler ou du Lauretis et elle nous a dit : " maintenant les filles on va être belles et intelligentes ». Et, tu vois, je n'avais jamais pensé que mon personnage allait autant gagner en complexité. Aujourd'hui je conseille à tous les bébés Drag qui arrivent de se nourrir de ça. Depuis on a tourné avec des lectures DragQueer, comme ça nous aussi on fait découvrir ces textes à notre public. On est des bibliothèques sur talons. On est folles $"$.

\footnotetext{
${ }^{14}$ Terme couramment employé pour désigner les autres Drag Queens, notamment celles qui appartiennent au même réseau relationnel.

${ }^{15}$ Il ne s'agit pas d'opposer un registre qui serait politique aujourd'hui avec un contexte sanitaire dans les années 1990. Bien évidemment, parler de VIH est politique. II s'agit plus particulièrement de situer les registres politiques dans deux temporalités aux revendications parfois divergentes. Lire par exemple : Broqua Christophe (2005), Agir pour ne pas mourir ! Act Up, les homosexuels et le sida. Paris, Presses de Sciences Po.

${ }^{16}$ Il s'agit ici de considérer le contexte français et non le contexte Drag Queen international qui, sur bien des aspects, a su mettre en avant de manière plus évidente l'aspect " politique » du Drag (Mémeteau, 2019 ; Kucharski, 2018).

${ }^{17}$ Expression pour qualifier le / la chef.fe d'une " house ».
} 
En creux de cette légitimité théorique et politique, un discours de scission entre les " bonnes » et les " mauvaises » Drag apparaît. Les Drag ne sont évidemment pas les seules à connaître ces effets de hiérarchisation par l'usage d'un vocabulaire adapté, par l'emploi de références théoriques reconnues, ou bien encore par l'inscription de leurs pratiques dans le champ des luttes politiques (en l'occurrence ici, c'est souvent la lutte contre les LGBTphobies qui est évoquée). Les univers de fans, tels que les décrit Florent Favard par exemple, sont eux aussi soumis aux labellisations de "vrai.e.s » et de "faux/sses » fans, c'est-à-dire de personnes légitimes ou illégitimes à s'exprimer sur ou par une culture (Favard, 2017). C'est ce que relève $K$. jeune Drag de province, qui voit d'un mauvais œil la nécessité du discours politique. "Je sais bien que lorsque tu ne dis pas " queer " ou "féminisme " c'est comme si tu n'avais pas le sésame pour être une Drag Queen. Moi je m'en moque. Ce dont j'ai envie c'est de créer un personnage et avec mes sisters on n'a pas pour prétention d'être armées de notes de bas de page. C'est un peu comme si on nous disait " choisis ta référence " alors que justement on essaie de déconstruire nos propres références. Y'a quelque chose d'injonctif à ça et je n'aime pas trop ça ". Etre Drag-queen, ce n'est donc pas qu'une expérience scénique de la théorie. Ce sont aussi des expériences biographiques, des expériences de vie. Il revient à chaque Drag de trouver son équilibre dans l'endroit émotionnel où elle se sent le mieux (Deluca, 2020 ; Verta, 2014). C'est ce sur quoi revient K. : « J'ai envie de prendre du plaisir surtout. Et c'est ça qui se voit lorsqu'on nous regarde : le plaisir qu'on a à danser, à rire, à jouer. C'est essentiel pour moi ».

\section{Ru Paul : une figure tutélaire mais controversée}

Si chaque communauté à sa figure tutélaire, celle des Drag Queens est indéniablement RuPaul. Encensé ou conspué, RuPaul est discuté dans l'ensemble des entretiens comme un élément de comparaison attractif ou répulsif en fonction des Drags (Simmons, 2014 ; Mémeteau, 2014; Edgar, 2011). RuPaul est une célèbre Drag Queen américaine, mondialement popularisée par sa téléréalité «RuPaul's Drag Race » qui est aujourd'hui diffusé sur Netflix et dont la première diffusion date du 2 février 2009. RuPaul est né le 17 novembre 1960 à San Diego et est également connue pour quelques succès musicaux et cinématographiques. Mais aux yeux du grand public, c'est indéniablement le RuPaul's Drag Race qui signe sa reconnaissance. "II y a un avant et un après RuPaul " pour V.Q. Elle précise : " en réalité c'est la dimension entertainement qui change tout. Avec ce show, avec Netflix, on entre dans les chaumières ". Si l'on assiste au passage d'une visibilité ciblée (dans les années 1990) à une augmentation réelle des scènes Drag en France comme dans d'autres pays, c'est non seulement que des figures Drag sont inoculées dans la culture, non seulement que renaît une culture Drag (liée à de nouvelle revendications) mais plus encore qu'une démocratisation Drag est en cours. En tant que visage emblématique d'une culture, RuPaul est aussi au cœur de controverses. Deux polémiques récurrentes ont fini par lasser quelques-unes des Drag rencontrées. La première renvoie à une somme de propos tenus par la star au sujet des personnes trans qui, selon lui, ne seraient pas admissibles à son concours du fait des opérations chirurgicales réalisées ${ }^{18}$. Pour RuPaul le Drag doit être une

\footnotetext{
${ }^{18}$ La présence de Drag trans dans la saison 2018 de l'émission semble initier un changement de ce côté-là.
} 
déconstruction du genre faite par des " hommes ". Mais dans une culture où la réception se fait principalement par des personnes LGBT, la question de l'assignation de la pratique Drag à la catégorie cisgenre passe mal. " Je ne sais pas mais elle tourne conservatrice ma Mother Ru [...] Tu peux pas avoir lutté toute ta vie contre l'homophobie et lâcher un truc transphobe comme ça. Surtout quand t'es Drag " rétorque P., une Drag Queen parisienne qui regarde néanmoins son émission. La désidentification avec RuPaul n'est pas chose aisée : d'une part elle reste une figure incontournable dans le paysage médiatique Drag et d'autre part elle transporte avec elle une partie de l'histoire des Drag en héritage. "Je n'ai pas divorcé avec RuPaul " poursuit P. " C'est juste que je distingue bien ce qu'il a pu être et ce qu'il est maintenant $"$.

Dans les entretiens réalisés, la question des relations sociales revient fréquemment. Ami.e.s ou membres de la famille, une grande partie est reliée à l'image Drag par RuPaul : "Quand j'ai dit à ma mère que je voulais être une Drag Queen, bon d'abord ça l'a pas étonné, mais ensuite je lui ai montré les émissions de RuPaul. Maintenant on les regarde ensemble » raconte W., 22ans. S'il est ce lien entre l'inter et l'extra communautaire, RuPaul compte pourtant d'autres polémiques à son actif.

En 2014, Strings Sabrina Strings et Long Bui examinent l'émission télévisée « RuPaul's Drag Race » et mettent l'accent sur la différence de traitement, par les Drag elles-mêmes et au sein du dispositif télévisuel, de la performance de genre et des questions raciales. Pour les chercheuses, si l'espace de l'ambivalent et du contradictoire sont bel et bien investis par les candidates, les questions relatives aux marqueurs raciaux ne semble pas réellement posées. Autrement dit, en se concentrant sur la saison trois la série, les auteurs montrent comment les Drag Queens subvertissent et jouent avec les notions de "réalité " et de "fiction " de genre, mais trouvent une impasse dans les discussions ouvertes sur la race. La mise en scène raciale de certaines concurrentes observées par les chercheuses semble laisser voir un antagonisme entre les personnages noirs et leurs homologues blancs (ou asiatiques soulignent-elles), malgré l'acceptation générale de la flexibilité des genres. Ainsi, la performance subversive du côté du genre fournit un site contrasté et un espace sémiotique complexe qui ne permet pas de traiter des questions sensibles de race ou d'ethnie, en particulier lorsque certaines formes de stéréotypes sont utilisées ou récompensées (perruques, maquillages sombres, accentuation des lèvres). Mais ces polémiques ne semblent pas avoir eu de la même façon un écho en France et outre-Atlantique. "J'ai entendu parler de ces questions, mais je ne fréquente presque que des Drag banches alors on n'est pas concernées de la même manière. Aux Etats-Unis c'est très fort ces questions de réappropriation culturelle et tout, mais en France, enfin dans le milieu Drag, je ne crois pas que ces questions se soient encore posées " dit O., Drag Queen parisenne. Elle poursuit : "Peut-être aussi parce que je ne connais pas de Drag qui utilisent ce registre dans leurs créations. On a déjà eu des polémiques comme ça en France, et forcément ça a dû en faire réfléchir quelques-unes, je ne sais pas, dans l'usage du fond de teint par exemple ". La transposition des controverses autour de RuPaul entre la France et les Etats-Unis n'est donc pas spontanée. Mais, à bien écouter les Drags, la rapidité des échanges numériques autour de leurs pratiques, ou bien autour du personnage du RuPaul, accélère le processus de comparaison. « Ca va bien finir par arriver de toute façon » conclut $O$. 


\section{Drag des villes / Drag des champs : quand la pratique Drag se décentralise}

Si les années 1990 comprenaient des spectacles Drag dans des boîtes et bars gay, les années 2010 sont à l'origine d'une structuration bien plus dense sur le territoire, une structuration moins dépendante des espaces communautaires et nocturnes gay, au bénéfice de collectifs Drag plus autonomes. De toute évidence, Paris, reste la capitale française des scènes Drags. Depuis le début des années 2010 on ne compte plus les rendez-vous ponctuels ou récurrents autour de personnages Drag. En 2019, chaque dimanche, un Bingo Drag attire par exemple plusieurs dizaines de personnes, plus de cent selon certains témoignages. Toutefois, depuis quelques années, des villes comme Lille, Lyon, Toulouse et plus récemment Bordeaux ont vu éclore des "maisons " de Drag. "Avant je partais à Paris le plus souvent possible pour assister à des shows Drag et moi-même j'ai pu m'y produire une ou deux fois. Mais ça avait un coût : le transport, l'hébergement.... Alors j'ai décidé, avec une bande copines, de créer mon propre groupe de Drag " déclare D.C, Drag marseillaise. En 2018, Bordeaux voit naître sa première "House " baptisée "La maison éclose ". Les premiers pas du groupe sont rapides. En fond, rejaillit une question centrale : une culture très parisienne a-t-elle sa place en province ? Ce n'est pas tant la question de la légitimité que celle de pertinence du choix territorial qui est alors posé. C'est par l'affirmative qu'ont répondu les membres de la Maison éclose qui, depuis, se produisent dans plusieurs lieux festifs et nocturnes de la capitale aquitaine. La décentralisation de la culture Drag marque une réelle différence avec les années 1990 qui, sinon dans quelques clubs, n'était pas parvenue à provoquer la constitution durable de "houses". Si l'on retrouve des personnages Drag dans les marches des fiertés de l'ensemble des villes françaises dès les années 2013-2014, d'autres initiatives plus institutionnelles étonnent. Aussi des municipalités iront même jusqu'à inscrire dans leurs programmations officielles des représentations de Drag. Ainsi la mairie de Bordeaux intègrera une performance de la " house " locale dans son programme 2018 de la quinzaine de l'égalité (un cycle d'évènements contre les discriminations).

Cette expansion en province crée littéralement une offre locale en termes de représentations LGBT (surtout gay, avouons-le) et encourage de nouvelle " houses " à se former. "Quand on a débuté on était seules et on se demandait comment ça allait fonctionner, si ça allait prendre, si des gens allaient être réceptifs. On a fait des petits clubs pour débuter, des soirées associatives, comme ça, sans vraiment savoir où on mettait les pieds, et très vite on a croulé sous les demandes. On peut performer devant plus de 500 personnes en une semaine. C'est inespéré. On a même entendu qu'une nouvelle maison allait se constituer. On a rien contre évidemment, il en faut pour tous les goûts et y'a de la place pour tout le monde dans le monde Drag " relate SH. Drag dans une grande ville de province.

Du côté du public, la demande ne semble pas se tarir. "Quand on pense "évènement LGBT ", on pense immédiatement à " Drag " " souligne un militant gay très actif dans un centre LGBT de province. "Y'a toujours eu des performers, des Drag Queens ou des spectacles dans les soirées de Pride ou dans d'autres évènements LGBT mais là on a des adhérent.e.s qui clairement nous demandent d'organiser des rencontres ou des soirées sur 
cette thématique " poursuit-il. Un nouveau paysage festif et politique local se dessine alors, avec des créatures que l'on croyait depuis quelques années désuètes ou ringardes. L'hypothèse d'une période "post mariage pour tous " qui aurait nécessité de réinventer les modes de visibilités et d'expressions LGBT (surtout gay encore une fois) se fait jour. "On assiste peut-être à une nouvelle page dans l'histoire de la visibilité et des soirées LGBT. On a tellement parlé mariage et on risque encore de tellement parler de PMA, que les gens ont tout simplement envie d'autre chose que de cortèges et de manifestations " conclut ce même militant. Cette hypothèse alimente le constat relatif à une augmentation récente des lieux et des visibilités Drag. Dans un souci d'oppositions locales aux Manifs pour tous, ellesmêmes marquées par des mises en scènes médiatiques puissantes, les Drag deviennent un dispositif offensif et festif de visibilité et de défense politique de la pluralité des genres et des sexualités.

\section{Vivre du Drag : la précarité au cœur des récits}

Parmi les multiples échanges avec ces Drag Queens réside une question transversale à toutes les expériences : celle de la précarité. Voilà très certainement un élément commun aux deux décennies ici mobilisées. A l'image des univers militants et associatifs (Swidzinski, Zamora-Cruz et Alessandrin, 2019), la précarité économique (comparée au temps d'investissement dans la constitution des scénarii, des costumes, de l'organisation etc...) revient très fréquemment. " $\mathrm{Si}$ tu comptes les costumes, le maquillage, les répétitions, les défraiements si tu es accompagnée etc. etc., tu ne t'en sors pas " selon J. Drag de 32ans « à la retraite ". "J'ai dû arrêter. A Paris tu paies pas ton loyer avec ce que tu gagnes en tant que Drag " poursuit-il. Alors, comme de nombreuses Drag il a longtemps alterné sa pratique Drag et un emploi « alimentaire ". "C'est ce qu'on fait toutes » rappelle-t-il. " II y en a peutêtre quelques-unes qui parviennent à vivre de leur art mais c'est rare ". Même si je ne les ai pas rencontrées, ces "quelques-unes" existent en effet peut-être car de nombreuses interviewées en font mention.

La précarité économique se mêle aussi de précarité relationnelle. Toutes les Drag n'échangent pas aussi facilement que $W$. et sa mère sur RuPaul. Alors, un « vrai » métier aux yeux de certains proches agit comme un paravent à d'éventuelles remarques ou critiques. "Je n'en ai pas parlé à mes parents » dit S. "Mes ami.e.s sont parfois au courant mais ça s'arrête là ". Les empêchements à raconter sa pratique en dehors des milieux habitués semblent de plusieurs ordres. D’une part, certaines Drag rêvent de vivre de leur pratique et, pour leurs proches, cette perspective professionnelle serait « inconcevable » pour reprendre le terme employé par S. Métier "indigne », "faux métier », métier " dangereux » : les représentations se bousculent lorsque les Drag imaginent ce que pourraient penser certains membres de leur famille à l'évocation de leur personnage. Mais il y a aussi tout ce que l'imaginaire Drag charrie comme préjugés à l'instar des amalgames entre " Drag Queens »"travestissement " et "transidentités » (Greco, 2016). "J'ai des ami.e.s qui pensent surement que comme je me Drag-queene je vais changer de sexe, ou quelque chose dans le genre. Mais ils n'osent pas vraiment $\mathrm{m}^{\prime}$ en parler. Je sais que ça les taraude mais ils gardent ça pour eux. Pour l'instant » déclare D.C. 
Si tous les témoignages évoquent la précarité économique, ils ne sont cependant pas tous unanimes sur les coupures relationnelles qu'engendrerait un "coming-out Drag " ${ }^{19}$. Pour certaines au contraire, vivre au grand jour c'est aussi assumer sa pratique auprès de sa famille par exemple. "Par contre, pour mon employeur je pense que ce serait compliqué " souligne R. de P. ; propos que confirment de très nombreux témoignages. La réputation Drag et le monde du travail ne semblent pas faire bon ménage. Lors de leur médiatisation dans des journaux télévisés ou des journaux papiers, certaines Drag relatent aussi cette crainte d'être reconnues. D'autres expériences, plus frontalement douloureuses, font état de discriminations. La journée M.B est enseignant dans une école supérieure privée. Un jour qu'il performait dans une boîte, et alors qu'il se pensait méconnaissable, il s'est confronté à un groupe d'étudiants qui l'ont identifié et ont diffusé des photos de la performance à l'ensemble de son établissement. M.B. n'a pas souhaité se défendre, et n'a d'ailleurs pas été défendu. "Aucun.e de mes collègue n'est venu m'en parler. J'étais devenu le paria de l'école " dit-il. A la suite de cet évènement, il a préféré démissionné et se chercher un autre poste. Dans ces circonstances, des émissions comme le RuPaul's Drag Race sont perçues par certaines comme une illusion. Pour M.P, RuPaul « c'est joli, ça fait rêver. Mais la vie d'une Drag française de province c'est pas les contrats de RuPaul pour Netflix ».

En creux de cette précarité et des épreuves (notamment discriminatoires) que la pratique Drag soulève, se pose la question de la notoriété et des réputations (Marzano, 2016). Dans un contexte de visibilité numérique, comme assurer simultanément une vie privée et une vie publique recherchée? Comment convertir une notoriété numérique dans provoquer d'irréputation ou de harcèlement en ligne (Beuscart et Meller, 2015) ? Pour ainsi dire, si la précarité et les discriminations semblent traverser les époques, un élément majeur distingue ici les deux époques: Internet, sa portée commerciale, son poids réputationnel et le cyberharcélement (Benedetto-Meyer et Klein, 2015).

\section{Conclusion : les nouvelles « mauvaises images ॥ de l'homosexualité}

La figure Drag reste donc un paradoxe : visibilisée dans les médias depuis quelques années, elle demeure majoritairement une figure de la nuit, principalement des nuits gays d'ailleurs. Pourtant la question de l'espace public revient fréquemment dans les témoignages comme une volonté d'une plus grande visibilité encore. Se réapproprier l'espace lors des marches des fiertés ou lors de manifestations est une question classique de la géographie du genre et des sexualités (Leroy, 2010). A l'instar des sœurs de la perpétuelle indulgence et des "folles» (Le Talec, 2013), les Drag seraient-elles devenues les nouvelles " mauvaises images " de l'homosexualité contemporaines, montrés du doigt car trop visibles et mises au centre des manifestations car attractives ? Quelle place sont-elles amenées à jouer dans les futures mobilisations publiques LGBT ? Plus encore, dans leur proximité avec le milieu gay, que provoquent les interactions entre les masculinités gays et la visibilité Drag (Berkowitz, Belgrave et Halberstein, 2007 ; Bishop, Kiss et al. 2014) ? Les interrogations que soulève ce

\footnotetext{
${ }^{19}$ L'expression « coming out Drag » est utilisée par certaines Drag et reprise dans quelques médias français comme étrangers pour stipuler l'instant de la révélation du personnage à des proches.
} 
renouveau Drag restent pour l'instant sans réponse. Et si l'on s'interesse aux Drag dans une perspective plus professionnelle, des questions relatives à la scène Drag française subsistent : que sont devenues les Drag des années 1990 ? Quelles carrières (au sens beckerien) anciennes et nouvelles Drag connaissent-elles? Les Drag actuelles sont pourtant à l'intersection de nombreux phénomènes sociaux et culturels, comme le fourmillement des identités de genre, des questions de fluidité de genre ou de la lutte contre les LGBT-phobies. Mais, à peine sédimentées, on ne sait si, comme leurs prédécesseuses des années 1990, elles parviendront à maintenir leurs communautés et leur visibilité.

\section{Bibliographie}

Alessandrin Arnaud (2021), Déprivilégier le genre : faire contre et être tout contre le genre, Double ponctuation ed.

Alessandrin Arnaud (2018), Sociologie des transientités, Cavalier Bleu ed.

Alessandrin Arnaud (2014), "Drag Kings : retour sur un atelier mixte », Miroir / Miroirs n.2, pp : 39-47.

Benedetto-Meyer, Marie, et Nicolas Klein. " Entre pratique commerciale et travail réputationnel : l'invention d'une nouvelle forme de relation client ? ", Terrains \& travaux, vol. 26 , no. 1,2015 , pp. $147-166$.

Berkowitz Dana, Belgrave Linda, Halberstein Robert (2007), « The Interaction of Drag Queens and Gay Men in Public and Private Spaces ", Journal of homosexuality, vol.52 n.3-4, pp : 1132.

Beuscart, Jean-Samuel, et Kevin Mellet. "La conversion de la notoriété en ligne. Une étude des trajectoires de vidéastes pro-am ", Terrains \& travaux, vol. 26, no. 1, 2015, pp. 83-104.

Bishop Christopher, Kiss Mark, Morrison Todd, Rushe Damien, Specht Jacqueline (2014), "The Association Between Gay Men's Stereotypic Beliefs About Drag Queens and Their Endorsement of Hypermasculinity ", Journal of homosexuality, vol.61, n.4, pp : 554-567.

Bressin Tiphaine et Patinier Jérémy (2012), Strike a pose : histoire(s) du voguing, Des ailes sur un tracteur.

Broqua Christophe (2005), Agir pour ne pas mourir ! Act Up, les homosexuels et le sida. Paris, Presses de Sciences Po.

Deluca Chrispher (2020), «Drag Queen Realness » pp : 184-187, Key concepts in curriculum studies (Judy Wearing et al. dir.), Routledge.

Edgar Eir-Anne (2011), «"Xtravaganza!": Drag Representation and Articulation in "RuPaul's Drag Race" ", Studies in Popular Culture, Vol. 34, n.1, pp : 133-146.

Edward Mark, Farrier Stephen (2020), Drag Histories, Herstories and Hairstories : Kings and Queens of Drag, Bloomsburry. 
Fassin Eric (2005), L'inversion de la question homosexuelle, Amsterdam.

Favard Florent (2017), "Crying theorizing, swooning: complexité narrative des séries télévisées et lectures (dé)genrées ", Fan studies et Gender studies : la rencontre (Bourdaa et Alessandrin dir.), pp : 85-100, Téraèdre

Foerster Maxime, Histoire des transsexuels en France, La musardine, 2012.

Greco Luca, et Stéphanie Kunert (2016). "Drag et performance ", Encyclopédie critique du genre. Corps, sexualité, rapports sociaux (Rennes Juliette dir.). La Découverte, 2016, pp. 222231.

Greco Luca (2014), " Tout ce que vous avez toujours voulu savoir sur les Drag Kings ", Miroirs / Miroirs n.2, pp : 27-39.

Kucharski, Kyle, (2018), The Gentrification of Drag, Mémoire de journalisme, CUNY Academic Works.

Leroy Stéphane (2010), " "Bats-toi ma sœur ». Appropriation de l'espace public urbain et contestation de la norme par les homosexuels », Métropoles [En ligne], n.8.

Marzano Michela. " Du désir de gloire à la quête de célébrité : réputation et reconnaissance ", Sens-Dessous, vol. 18, no. 2, 2016, pp. 57-66.

Mèmeteau, Richard (2019), Pop culture: Réflexions sur les industries du rêve et l'invention des identités, La Découverte

Mémeteau Richard (2014), "RuPaul : éthique du commérage ", Miroir / Miroirs, n.2, pp : 95104.

Patinier Jeremy (2014), « Typologie de la Drag Queen », Miroir / Miroirs, n.2, pp : 81-87.

Perreau, Bruno (2018). Qui a peur de la théorie queer ?. Paris: Presses de Sciences Po.

Roth-Bettoni Didier (2007), L'homosexualité au cinéma, La musardine.

Roth-Bettoni Didier (2016), "Sisi Imperator ", Les cahiers de la transidentité vol.3, "Corps trans Corps queer $"$, Alessandrin et al. dir. 159-169.

Schacht Steven, Underwood Lisa (2003), The Drag queen anthology : the absolutely fabulous but flawless customary world of female impersonators, Harrington Press.

Shaffauser Thierry, (2014) « Drag Queen Feminism », Miroir / Miroirs, n.2, pp : 87-94.

Simmons Nathaniel (2014), "Speaking Like a Queen in RuPaul's Drag Race: Towards a Speech Code of American Drag Queens ", Sexuality \& Culture, vol.18, n.3, pp : 630-648.

Strings Sabrina, Bui Long (2014), " "She Is Not Acting, She Is » : The conflict between gender and racial realness on RuPaul's Drag Race " Feminist Medis Studies, vol.14, n.5, pp : 822-836.

Swidzinski Jeanne, Zamora-Cruz Clémence, Alessandrin Arnaud (2019), "Militantisme trans et précarité ", Actualité des trans studies, pp : 77-85, Editions des Archives Contemporaines. 
Talec Le Jean-Yves (2013), "L'espace des folles ", Géographie des homophobies (Raibaud et Alessandrin dir.), pp : 225-240, Armand Colin.

Talec Le Jean-Yves (2008), Folles de France: Repenser I'homosexualité masculine, La Découverte.

Verta Taylor, Leila Rupp (2015), "When the Girls Are Men: Negotiating Gender and Sexual Dynamics in a Study of Drag Queens ", Journal of Women in Culture and Society vol. 30, no. 4, pp : 2115-2139. 